\title{
Isolation, expression and function analysis of a bZIP transcription factor IbbZIP37 in sweetpotato (Ipomoea batatas L. [Lam])
}

\author{
Wenbin Wang ${ }^{1 *}$, Xiangpo Qiu', Yanxin Yang ${ }^{2}$, Ho-Soo Kim³ ${ }^{3}$ Xiaoyun Jia ${ }^{1}$, Huan Yu1, Sang-Soo Kwak ${ }^{\text {* }}$ \\ ${ }^{1}$ College of Life Science, Shanxi Agricultural University, Taigu 030801, China, ${ }^{2}$ College of Arts and Science, Shanxi Agricultural University, \\ Taigu 030801, China, ${ }^{3}$ Plant Systems Engineering Research Center, Korea Research Institute of Bioscience and Biotechnology (KRIBB), \\ 125 Gwahak-ro, Daejeon 34141, South Korea
}

\section{A B S T R A C T}

\begin{abstract}
bZIP transcription factor play an important regulatory role in the response to multiple abiotic stresses. However, our knowledge of the stress tolerance functions of bZIP family genes in sweetpotato (Ipomoea batatas [L.] Lam) remains limited. In the present study, we isolated and functionally characterized an IbbZIP37 gene encoding an abiotic stress-inducible bZIP group A transcription factor. Sequence analysis showed that the IbbZIP37 contained a typical bZIP domain and five conserved Ser/Thr kinase phosphorylation sites (RXXS/T). The IbbZIP37 protein was localized in the nucleus and possessed transcriptional activation activity. The results of electrophoretic mobility shift assays indicated that IbbZIP37 can bind to the ABRE cis-element, not do to DRE cis-element in vitro. The IbbZIP37 gene showed the highest level of constitutive expression in root, especially in fibrous root and storage root body. Gene expression was induced by ABA and several environmental stresses including drought, salt and heat shock. Our results suggest that IbbZIP37 is a positive transcription regulator of the abiotic stresses response, which can be used as an excellent candidate for improving the stress tolerance of different crop plants.
\end{abstract}

Keywords: Abiotic stress tolerance; Abscisic acid; bZIP; Sweetpotato

\section{INTRODUCTION}

Sweetpotato (Ipomoea batatas [L.] Lam) has the seventh highest annual production worldwide among food crops (Pradhan et al., 2015). Sweetpotato is considered a health food because of the high content of dietary fiber, carotenoids, vitamins, carbohydrates, and minerals. Furthermore, sweetpotato is widely used as a rich source of starch and bioethanol (Madzlan et al., 2012; Duvernay et al., 2013). However, pests, viral diseases, and various environmental stresses such as drought, high salinity, extreme temperature, and variable climates generally limit the production of sweetpotato in many areas worldwide (Lebot, 2010). Understanding the mechanisms involved in the activation of adaptive stress responses is essential for the development of transgenic strategies to improve stress tolerance in sweetpotato.

The plants evolved a number of defense mechanisms in response to adverse environment change. Transcription factors (TFs) play an important regulatory role by activating their target gene expression following with binding to the conserved cis-acting elements, and this leads to improved stress tolerance (Fujita et al., 2005). Numerous stress responsive TFs including bZIP, MYB, WRKY, bHLH and NAC families have been well revealed through genetic, molecular and biochemical analyses (Wang et al., 2003; Golldack et al., 2011). Overexpression of these TFs have marked effects on plant growth, stress tolerance, and photosynthetic activity (Hossain et al., 2010a).

As one of the largest TF groups, basic leucine zipper (bZIP) protein contains a basic region that binds DNA and a leucine zipper dimerization motif (Latchman, 1997). Recent studies have shown that bZIP TFs are involved in multiple diverse biological processes such as seed germination, flower and seed development, biotic and abiotic stress responses, and hormone and sugar signalling (Thurow et al., 2005; Muszynski et al., 2006; Lindemose

\footnotetext{
*Corresponding authors:

Wenbin Wang, Ph.D., College of Life Science, Shanxi Agricultural University, Taigu 030801, China. E-mail: sxndwwb@126.com

Sang-Soo Kwak, Ph.D., Plant Systems Engineering Research Center, Korea Research Institute of Bioscience and Biotechnology (KRIBB),

125 Gwahak-ro, Daejeon 34141, South Korea. Tel.: +82-42-860-4432. E-mail: sskwak@kribb.re.kr
}

Received: 01 January 2019; $\quad$ Accepted: 28 February 2019 
et al., 2013). Generally, bZIP TFs can be classed with ten groups including group $\mathrm{A} \sim \mathrm{I}$ and $\mathrm{S}$ in Arabidopsis based on sequence similarity of the basic region and additional conserved motifs (Jakoby et al., 2002). ABA-response element binding protein (AREB), also referred to as ABRE-binding factor (ABF), belong to Group A of bZIP family. Five conserved Ser/Thr kinase phosphorylation sites (RXXS/T) are the characteristic of the abiotic stressresponsive $A t A B F s / A t A R E B s$ as previously reported (Furihata et al., 2006; Fujii et al., 2009). In addition, the (C/T)ACGTGGC consensus core sequence has been identified as the major cis-acting regulatory element (Hirayama and Shinozaki, 2010; Yoshida et al., 2010).

AREBs can regulate the expression of $\mathrm{ABA}$ and/or stress-response genes by binding their ABRE which normally locates in the promoter regions (Busk and Pagès, 1998; Hyungin et al., 2000). AREB subfamily genes have been extensively investigated in a number of plant species, such as Arabidopsis, rice, wheat, barley, potato and tomato (Hyungin et al., 2000; Casaretto and Ho, 2005; Kobayashi et al., 2008; Hossain et al., 2010b; Tsaihung et al., 2010; García et al., 2012). Most AREBs such as bZIP36/AREB1/ABF2, bZIP37/ABF3 and bZIP38/AREB2/ABF4 are highly induced by ABA, drought and salinity treatments in plant tissues (Uno et al., 2000; Furihata et al., 2006; Yoshida et al., 2010). Furthermore, overexpression of AREBs could increase abiotic stress tolerance in plant species (Hossain et al., 2010b; Roychoudhury et al., 2013). For example, overexpressing of bZIP37 / ABF3 or bZIP38 / AREB2 / ABF4 in Arabidopsis showed improved drought tolerance via the up-regulation of several ABA/stress-responsive genes (Kang et al., 2002). Alfalfa overexpressing AtbZIP37/AtABF3 gene could reduce transpiration rate, decrease reactive oxygen accumulation and improve stress tolerance to drought, salt and oxidative stress (Wang et al., 2016b).

In present study, a sweetpotato IbbZIP37 gene encoding an abiotic stress-inducible bZIP group A transcription factor was isolated and functionally characterized. The transactivation and ABRE-binding ability of IbbZIP37 was then tested using a yeast system and electrophoretic mobility shift assays (EMSA). The expression pattern of IbbZIP37 in different tissues and in the response to ABA and several environmental stresses was also evaluated. Our results indicated that IbbZIP37 is a positive transcription regulator of the abiotic stresses response, which can specifically bind to the ABRE.

\section{MATERIALS AND METHODS}

\section{Isolation of the IbbZIP37 gene}

Sweetpotato (cv. Xushu 18) was used in this study. Sweetpotato plants were propagated by cutting and grown for three weeks at $25 \pm 1^{\circ} \mathrm{C}$ under $16 \mathrm{~h}$ light $/ 8 \mathrm{~h}$ dark photoperiod. The full-length open reading frame (ORF) of IbbZIP37 was amplified from total RNA by reverse transcription PCR (RT-PCR) using gene-specific primers (Table 1). The PCR product was cloned into the T-blunt vector (BioFACT, Daejeon, Korea) and sequenced to confirm the fidelity.

\section{Bioinformatic analysis of the IbbZIP37 gene}

AtbZIP TFs amino acid sequences were downloaded from the National Center for Biotechnology Information (NCBI) (http://blast.ncbi.nlm.nih.gov/Blast.cgi). To investigate the relationship between IbbZIP37 and AtbZIP TFs from $A$. thaliana, a systematic phylogenetic analysis was performed using the neighbor-joining method in the MEGA (version 5.1) software with 1,000 bootstrap replicates. The amino acid sequences of several bZIP proteins were compared using DNAMAN software (Version 5.2.2.0, Lynnon Biosoft, USA), and highly conserved amino acid residues were analyzed using the SMART program (http://smart.embl-heidelberg.de/).

\section{Subcellular localization of IbbZIP37}

The full-length cDNA of IbbZIP37 was fused into pGWB5 vector containing the green fluorescent protein (GFP) tag under the control of the CaMV $35 S$ promoter using the gateway cloning method (Curtis and Grossniklaus, 2003). The fusion vector $p 35$ S: IbbZIP37-GFP was transformed into Agrobacterium tumefaciens strain GV3101, which was then used for transient transformation of tobacco (N. benthamiana) leaves. The transformed $N$. benthamiana leaves were cultivated after 3 days and subjected to GFP fluorescence analysis under confocal laser scanning microscope (Leica Microsystems, Heidelberg, Germany). Cells were labeled with the DNA dye 4,6-diamidino-2phenylindole (DAPI) to visualize the nucleus.

Table 1: Primers used for PCR analysis

\begin{tabular}{lll}
\hline Primer name & Primer sequence (5' to 3') & Application \\
\hline IbbZIP37-F & ATGATGGGGTCATACTTGGA & Vector construction \\
IbbZIP37- $R$ & TTACCAAGGCCCAGTAAGCG & Vector construction \\
IbbZIP37-RT-F & CAGCTGAATAGTATGGGATT & Real-time PCR \\
IbbZIP37-RT-R & CTGGTGGCTCCTACACTAAG & Real-time PCR \\
IbActin- $F$ & GTTATGGTTGGGATGGGACA & Real-time PCR \\
IbActin- $R$ & GTGCCTCGGTAAGAAGGACA & Real-time PCR \\
\hline
\end{tabular}




\section{Transcriptional activation activity of IbbZIP37}

The full-length sequences of IbbZIP37 and AtbZIP37 (positive control) cDNA were fused in-frame with the GAL4 DNA binding domain in the pDEST32 vector using the gateway cloning method. The fused constructs pDEST32-IbbZIP37 and pDEST32-AtbZIP37, as well as negative control $p D E S T 32$ empty vector were transformed individually into yeast strain PJ69-4A containing the His3 and $L a c Z$ reporter genes. Transformed yeast cells were cultured on synthetic-defined (SD) plates containing $\mathrm{SD} / \mathrm{Leu}^{-}$, and $\mathrm{SD} / \mathrm{Leu}^{-} / \mathrm{His}^{-}$media. The transactivation activity of each protein was evaluated by filter lift assay and quantitative assays of $\beta$-galactosidase activity using O-nitrophenyl- $\beta$-d-galactopyranoside (ONPG). The $\beta$-galactosidase activity at $\mathrm{OD}_{420}$ was expressed in Miller units. All procedures were performed according to the Yeast Protocols Handbook (Clontech, USA).

\section{Purification of recombinant protein and electrophoretic mobility shift assays (EMSA)}

The full-length cDNA of IbbZIP37 was fused into pDEST15 vector containing the GST tag to generate GST: IbbZIP37 plasmid, and then was expressed in E. coli strain BL21. The recombinant protein was purified as described (Jin et al., 2017). Optimal conditions for fusion were created by adding $0.4 \mathrm{mM}$ isopropyl- $\beta$-d-thiogalactoside (IPTG), and incubating the E. coli at $20^{\circ} \mathrm{C}$ for $12 \mathrm{~h}$. Oligonucleotide sets containing the ABRE repeat element (5'-GGACACGTG GCGGGACACGTGGCGGGACACGTGGCG-3') or the DRE/CRT repeat element (5'-TTGATA CTACCGACATGAGTTGATACTACCGACAT GAGTT-3') were annealed by boiling for $5 \mathrm{~min}$ and labeled with $\left[\gamma^{-32} \mathrm{P}\right]$-ATP by adding T4 Polynucleotide Kinase (Promega, WI). A mixture of a $0.5 \mu \mathrm{g}$ labeled probe and $10 \mu \mathrm{g}$ purified GST-IbbZIP37 fusion protein or GST protein was incubated in binding buffer $(200 \mathrm{mM}$ HEPES, $5 \mathrm{mM}$ DTT, $1 \mathrm{mM}$ EDTA, $50 \mathrm{mM} \mathrm{KCl}$, and 20 pmol of poly dI-dC) at room temperature for $30 \mathrm{~min}$. And then the reaction mixture was loaded onto an $8 \%$ native polyacrylamide gel and visualized by autoradiography.

\section{Quantitative real-time PCR (qRT-PCR) of gene expression} Tissue-specific expression of IbbZIP37 was examined in various tissues (leaves, petioles, stems, fibrous roots, pencil roots, proximal end of storage roots, storage root bodies, and distal end of storage roots) of 10-week-old sweetpotato plants under no environmental stress. To examine the expression of IbbZIP37 under different abiotic stresses, sweetpotato plants were subjected to $10 \mu \mathrm{M} \mathrm{ABA}$, $25 \%$ polyethylene glycol (PEG8000), $350 \mathrm{mM} \mathrm{NaCl}$, or $47^{\circ} \mathrm{C}$ heat stress, and the third leaf from the top of sweetpotato plants was sampled at $0,3,6,12,24$, and $48 \mathrm{~h}$ post-treatment for analyzing IbbZIP37 gene expression by qRT-PCR.
Total RNAs and cDNA templates were obtained as previously described (Jin et al., 2017). All qRT-PCR analyses were performed in triplicate with a CFX RTPCR system (Bio-Rad, CA, USA) using Ever-Green PCR master mix kit (BioFact, Daejeon, Korea) according to the manufacturer's instructions. Relative expression of the detected gene was calculated using the $2^{-\Delta \Delta C T}$ method. The specific gene primer sequences for qRTPCR are listed in Table 1. The IbActin gene was used as an internal control.

\section{Statistical analysis}

Data were statistically analyzed using paired Student's $t$-test in SPSS Statistics 17.0 software (IBM China Company Ltd., Beijing, China) and Microsoft Excel. Measurements are shown as the mean \pm standard deviation (SD). All experimental assays used to obtain the results were repeated at least three times.

\section{RESULTS}

\section{Isolation and structural analysis of IbbZIP37}

The full-length cDNA sequence of IbbZIP37 gene was isolated from Xushu 18 and sequenced to be $1245 \mathrm{bp}$ in length with a complete open reading frame of 414 amino acids (Fig. 1A). The relationship between IbbZIP37 and bZIP group $\mathrm{A} \sim \mathrm{I}$ and $\mathrm{S}$ TFs from $A$. thaliana was analysed by MEGA 5.1. The result confirmed that IbbZIP37 was most closely to AtbZIP37 (Fig. 1B). Sequence analysis of the deduced amino acid residues using the DNAMAN and SMART program revealed that the protein contained a typical basic region leucine zipper (bZIP) domain (N-X7-R/K-X9-L-X6-L-X6-L), which included a basic DNA binding domain and a leucine zipper domain (Fig. 1C). Five conserved Ser/Thr kinase phosphorylation sites (RXXS/T) $(\square \sim \square)$ were also observed in IbbZIP37.

\section{IbbZIP37 was localized to the nuclei}

We constructed the transient expression vector including the fusion protein $p 35 \mathrm{~S}$ : IbbZIP37-GFP (Fig. 2A) and checked the subcellular localization of IbbZIP37 by transient expression in epidermal cells of tobacco (N. benthamiana) leaves. As shown in Fig. 2B, co-localization of green fluorescence with the DAPI signal indicated that IbbZIP37 protein was localized to the nuclei.

\section{IbbZIP37 exhibited transcriptional activation capability} We further determined whether IbbZIP37 could act as a transcriptional activator using the yeast one-hybrid system. The full length IbbZIP37 and AtbZIP37 were cloned respectively into a yeast expression vector $p D E S T 32$ which contains the GAL4 DNA binding domain. 


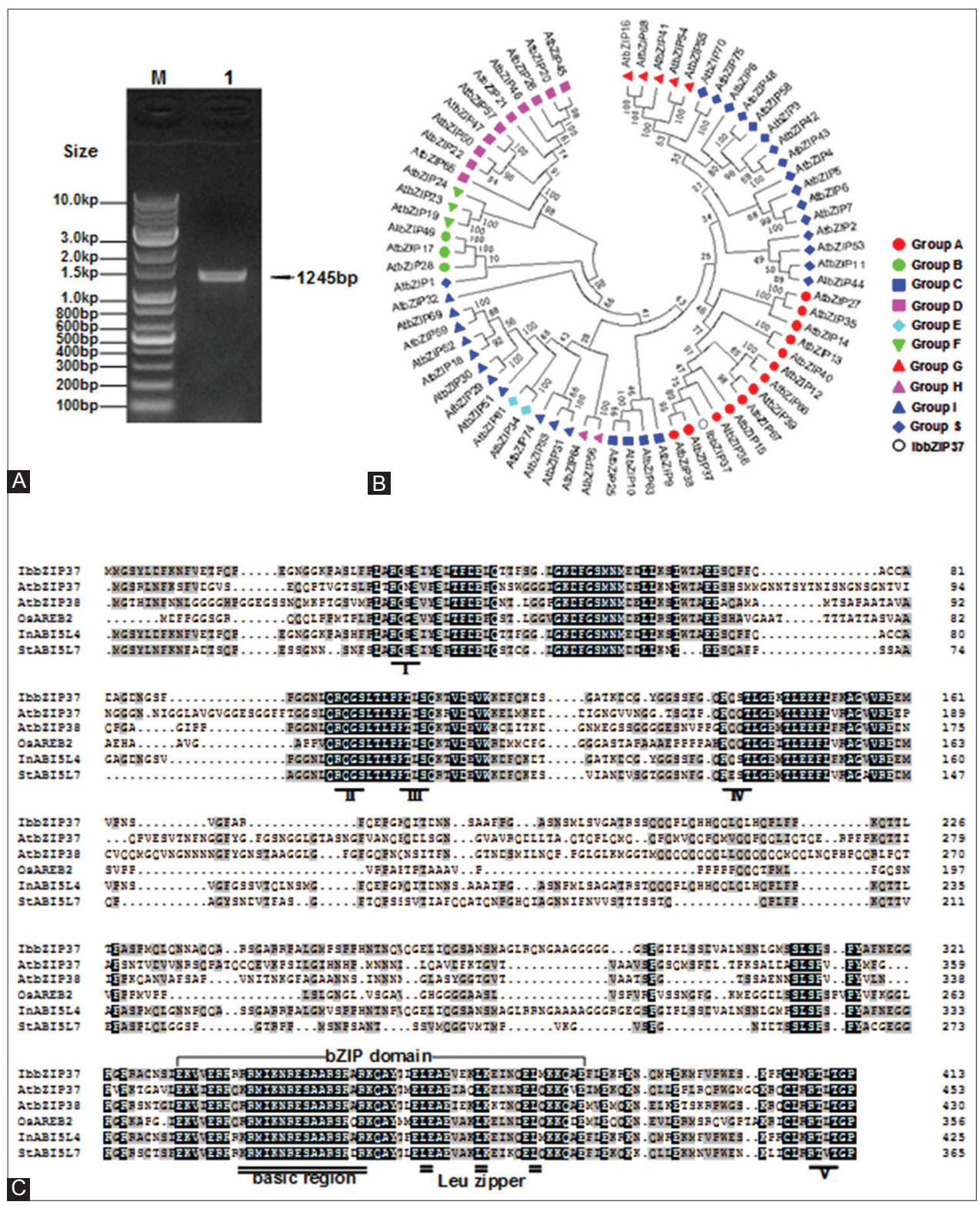

Fig 1. Isolation and bioinformatic analysis of the IbbZIP37 gene. A, Isolation of the IbbZIP37 gene; B, Phylogenetic relationship in IbbZIP37 with bZIP TFs of $A$. thaliana; C, Multiple sequence alignment of the conserved bZIP-domains and five Ser/Thr kinase phosphorylation sites in selected bZIP-related proteins. Amino acids shaded by color are conserved, with black indicating the highest similarity and gray less.

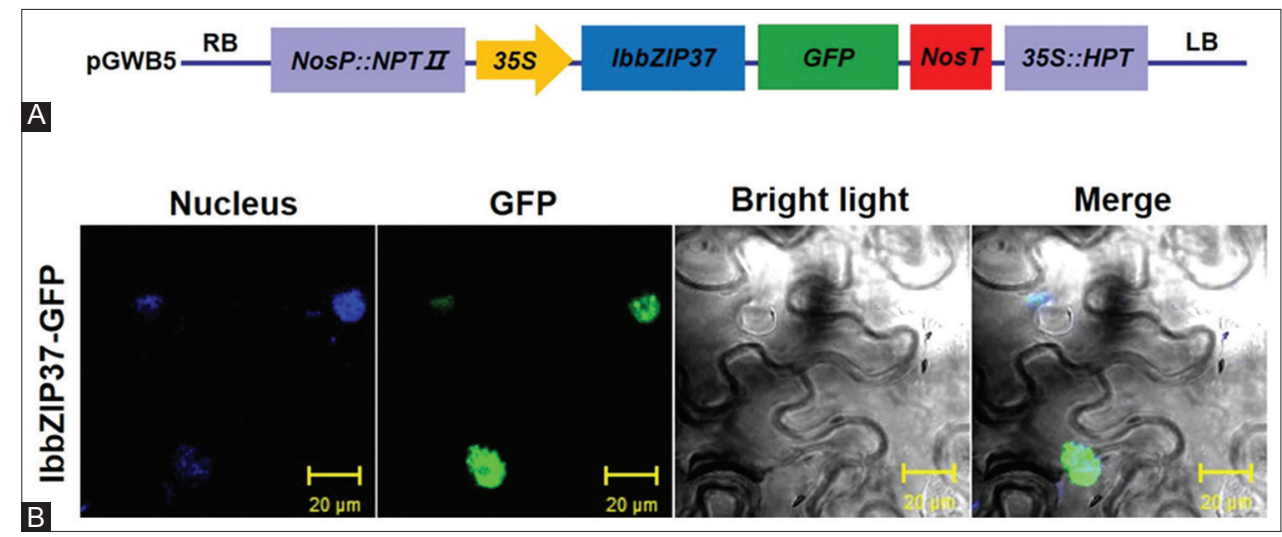

Fig 2. Subcellular localization of IbbZIP37 protein. A, Schematic representation of the expression vector construct used for tobacco transformation; B, Subcellular localization of IbbZIP37 protein. 


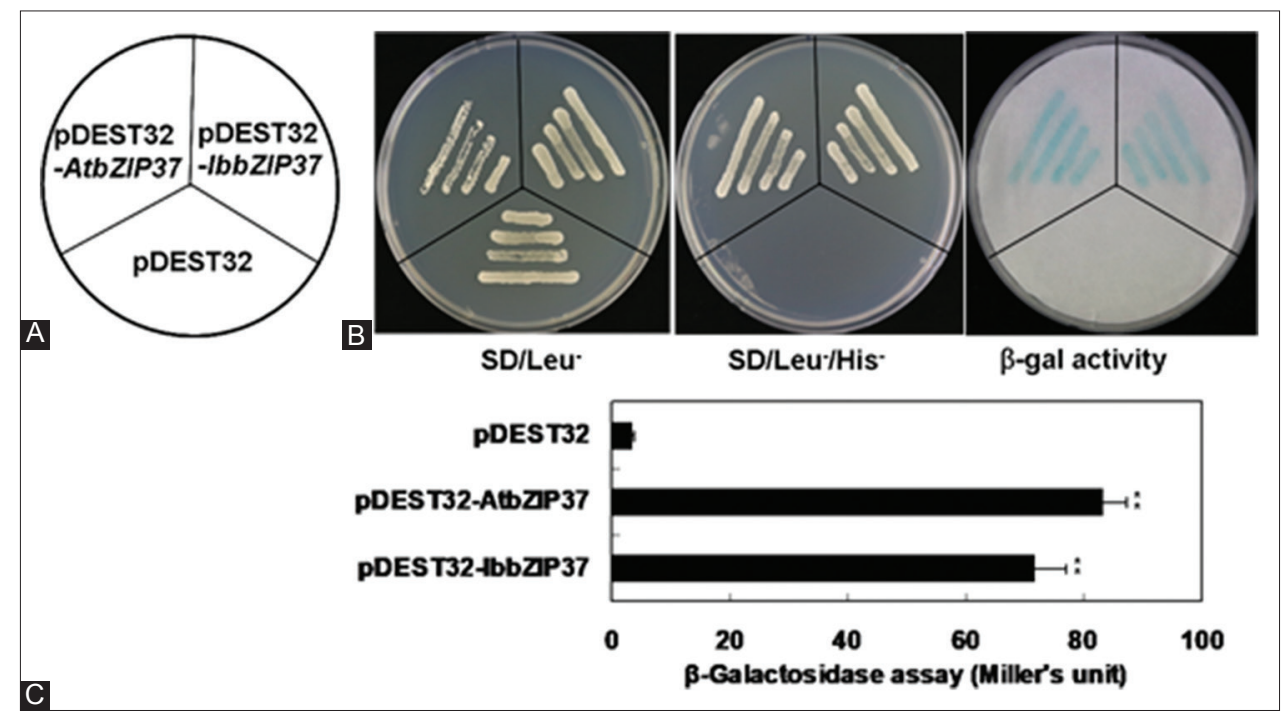

Fig 3. Transactivation activity assay of the IbbZIP37 protein. A, Transactivation activity assay of the IbbZIP37 protein. pDEST32-AtbZIP37 and pDEST32 empty vector were used as a positive and a negative control, respectively; C, Relative quantitative assay of $\beta$-galactosidase activity. $\beta$-galactosidase activity is expressed in Miller units. The error bars represent SD of three independent experiments. Asterisks indicate a significant difference compared with leaves at ${ }^{*} P<0.01$.

pDEST32-AtbZIP37 and pDEST32 empty vector were used as a positive and a negative control, respectively. All transformants grew well on SD/Leu plates as shown in Fig. 3A. Yeast cells harboring pDEST32-AtbZIP37 and pDEST32-IbbZIP37 grew normally on SD/Leu / His ${ }^{-}$medium and showed $\beta$-galactosidase activity, whereas yeast cells containing $p D E S T 32$ failed to grow on the $\mathrm{SD} /$ $\mathrm{Leu}^{-} / \mathrm{His}$ - medium and lacked $\beta$-galactosidase activity. These results revealed that IbbZIP37 possesses transcriptional activation capability. A relative quantitative assay of $\beta$-galactosidase activity using ONPG as substrate provided similar results (Fig. 3B).

\section{IbbZIP37 protein specifically binds to the ABRE cis- element in vitro}

ABA-responsive element (ABRE), often shared as a consensus in the promoters of $\mathrm{ABA}$ or stress regulated genes, can bind with the AREB/ABF protein. In order to determine whether IbbZIP37 binds to ABRE or DRE cis-elements in vitro, we performed EMSA using recombinant GST-IbbZIP37 and the ${ }^{32} \mathrm{P}$ labeled DNA containing $3 \times \mathrm{ABRE}$ or $2 \times \mathrm{DRE} / \mathrm{CRT}$ core element (Fig. 4A). Recombinant GST-IbbZIP37 was IPTG-induced expressed in E. coli BL21, and successfully purified by affinity chromatography (Fig. 4B). As shown in Fig. 4C, the shifted bands were observed in EMSA of ABRE and the binding activity was strengthened following with the more amounts of GST-IbbZIP37 protein (Fig. 4C, lane 3,4), whereas GST alone could not bind to labeled ABRE (Fig. 4C, lane 2). In addition, no shifted bands were observed in EMSA of DRE/CRT. These results indicated that IbbZIP37 protein was able to specifically bind to the ABRE cis-element in vitro.

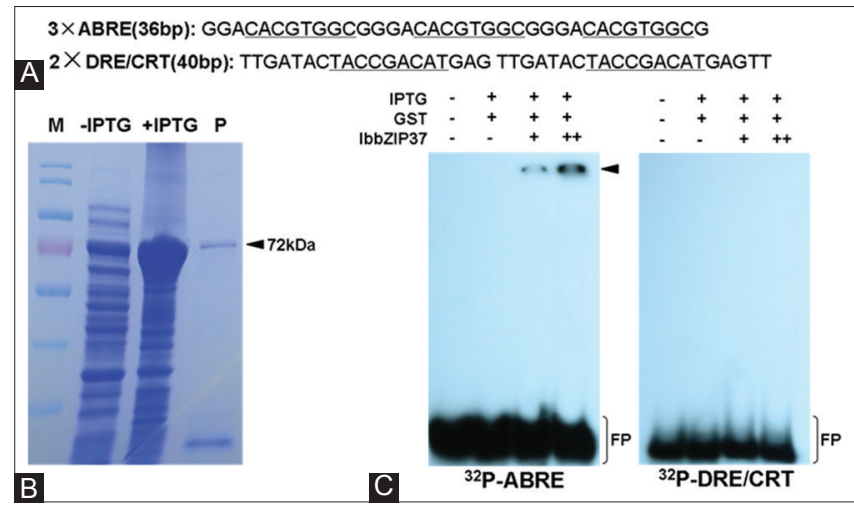

Fig 4. EMSA of IbbZIP37 binds with ABRE or DRE cis-element. $A$, Oligonucleotide sequences containing the ABRE or DRE repeat element were used as a probe in EMSA. Letters with underline indicate the core sequence; B, Purification of GST-IbbZIP37 fusion protein. $\mathrm{P}$ indicates the purified protein; $\mathrm{C}$, Analysis of binding specificity with cis-element. FP indicates free probe. - and + indicate no and addition of agent, respectively. ++ indicate increasing amounts of GST-IbbZIP37 protein $(10 \mu \mathrm{g})$ used for the DNA-binding analysis.

\section{Tissue-specific and stress-induced expression patterns of IbbZIP37 gene in sweetpotato}

The expression levels of IbbZIP37 were mensurated via qRTPCR in a range of tissues (Fig. 5A). IbbZIP37 was expressed in all of the analyzed tissues, and was higher in root, especially in fibrous root and storage root body (Fig. 5B).

The relative expression levels of IbbZIP37 gene in sweetpotato plants exposed to ABA, PEG, NaCl, and high temperature were also tested using the qRT-PCR method. As shown in Fig. 6, relative expression levels of IbbZIP37 began to increase after exposure to $10 \mu \mathrm{M} \mathrm{ABA}$ for $1 \mathrm{~h}$ and reached a peak at $3 \mathrm{~h}$, when levels were 3 -fold that of the non-treated control. And then it decreased 


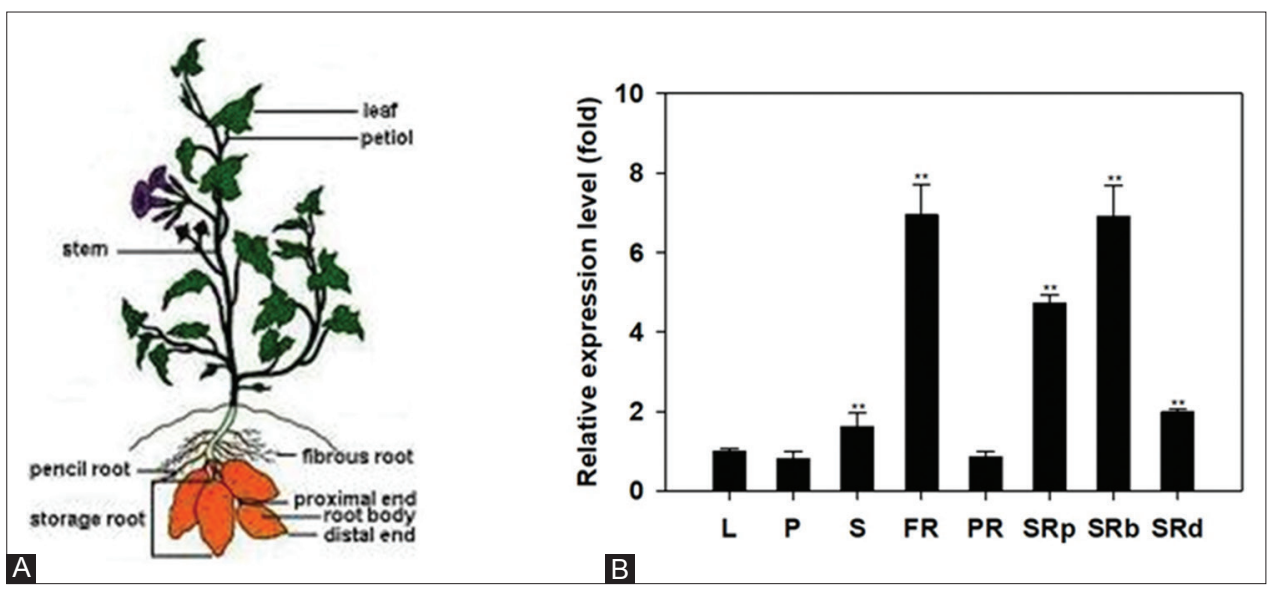

Fig 5. Tissue-specific expression of IbbZIP37 gene in sweetpotato. A, Morphology of sweetpotato plants; B, Tissue-specific expression analysis of IbbZIP37. L, leaf; P, petiol; S, stem; FR, fibrous root; PR, pencil root; SRp, proximal end of storage root; SRb, storage root body; SRd, distal end of storage root. Asterisks indicate a significant difference compared with leaves at ${ }^{* *} \mathrm{P}<0.01$ by t-test.

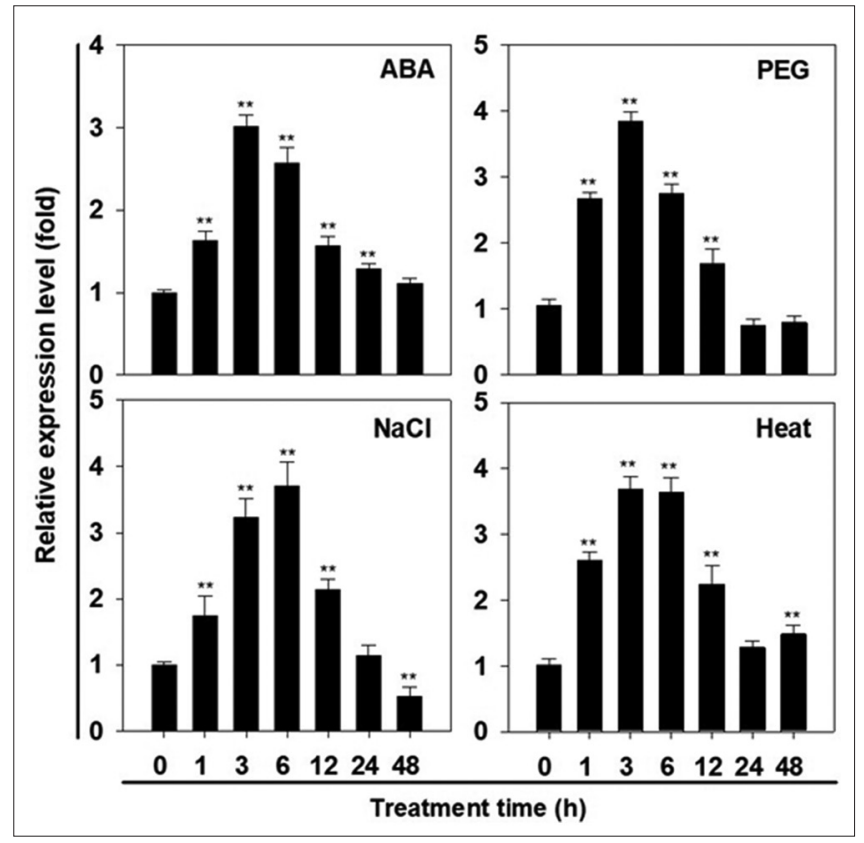

Fig 6. Expression patterns analysis of IbbZIP37 in response to abiotic stress. Three-week-old sweetpotato plants were subjected to ABA $(10 \mu \mathrm{M})$, dehydration (25\% PEG8000), high salinity (350 mM NaCl), and heat shock $\left(47^{\circ} \mathrm{C}\right)$ treatments. The relative expression level of the third intact fully expanded leaves (from the top) was measured by qRTPCR analysis The sweetpotato IbActin gene was used as an internal control. The error bars represent the mean \pm SD of three biological replicates. Asterisks indicate a significant difference compared with Oh at ${ }^{* *} \mathrm{P}<0.01$ by t-test.

over the following $3 \mathrm{~h}$. The expression of IbbZIP37 were also strongly induced by $25 \% \mathrm{PEG}, 350 \mathrm{mM} \mathrm{NaCl}$, and $47^{\circ} \mathrm{C}$ heat shock, and the similar expression pattern was observed. Under dehydration, salinity and heat stress, mRNA accumulation reached their highest levels at $3 \mathrm{~h}$ or $6 \mathrm{~h}$ with an up-regulation of 3.8-, 3.5- and 3.5-fold as compared to the control, respectively. Taken together, these results suggested that IbbZIP37 was induced by ABA, dehydration, high salinity and heat shock.

\section{DISCUSSION}

Sweetpotato is not only a nutrient-rich health food, but also a useful source of starch and bioethanol (Duvernay et al., 2013; Pradhan et al., 2015). However, abiotic stresses including drought, salt, and high temperature limit the production of sweetpotato, and then cause significant losses in yield (Lebot, 2010). The expression and function of genes regulating abiotic stress responses in sweetpotato are of considerable interest. To date, several important stress-related genes, including IbLCY- $\beta$ (Kim et al., 2014), IbOr (Sun et al., 2013; Goo et al., 2015; Kang et al., 2017b), IbPsbP (Kang et al., 2017b), IbCHY- $\beta$ (Kang et al., 2017a), $I b C B F 3$ (Jin et al., 2017), IbMPK3 and IbMPK6 (Kim et al., 2016) genes have been coloned from sweetpotato and functionally characterized in our laboratory. Previously, some bZIP genes have been reported with roles in response to abiotic stresses. However, there are few reports on the bZIP genes in sweetpotato. In the current study, we cloned a group A bZIP gene, IbbZIP37, and demonstrated its roles in adaptation to drought, salt and high temperature stresses.

Generally, the bZIP TFs have been divided into 10 Jakoby et al., 2002) or 13 (Corrêa et al., 2008) different groups in A. thaliana or rice (Oryza sativa), respectively. The basic DNA binding domain containing an invariant (N-X7$\mathrm{R} / \mathrm{K}$ ) motif and a leucine zipper domain (L-X6-L-X6-L). Previously reports showed that five conserved Ser/Thr kinase phosphorylation sites (RXXS/T) are the structure characteristic of the abiotic stress-responsive function in bZIP group A (Furihata et al., 2006; Fujii et al., 2009). In our results, IbbZIP37 also contained the typical basic region leucine zipper domain and the five conserved Ser/ Thr kinase phosphorylation sites (Fig. 1C). Consistently, amino acid sequence alignments demonstrated that IbbZIP37 is similar to other bZIP group A proteins 
including AtbZIP37, AtbZIP38, OsAREB2, InABI5L4 and StABI5L7. Together with the phylogenetic analysis (Fig. 1B), these findings suggested IbbZIP37 protein may be a functional homolog in response to abiotic stresses.

Our current study indicated that IbbZIP37 protein is localized to the nuclei (Fig. 2B). Yeast one-hybrid analysis also revealed that IbbZIP37 possesses transcriptional activation capability (Fig. 3). These results confirmed the basic characteristics of IbbZIP37 as a transcription factor. Previous yeast hybrid assay with OsABF1 (Hossain et al., 2010a), OsABF2 (Hossain et al., 2010b), OsbZIP23 (Xiang et al., 2008), and TaAREB3 (Wang et al., 2016a) have also shown that their respective $\mathrm{N}$-terminal regions control the transactivation activity. However, transcription regulatory regions of IbbZIP37 protein need to be further revealed. As a cis-acting element, ABRE is known as an important molecular switch involved in the transcriptional regulation of a dynamic network of genes (Yamaguchishinozaki and Shinozaki, 2005; 2006). Generally, AREB can regulate the expression of $\mathrm{ABA} /$ stress-response genes by binding their ABRE (Busk and Pagès, 1998; Hyungin et al., 2000). The results of EMSA experiments indicate that IbbZIP37 protein was able to specifically bind to the ABRE sharing the CACGTGGC consensus core sequence in vitro (Fig. 4C). Similar observations of AtABF1, SIAREB, TabZIP60, TaAREB3, and TabZIP14-B have been reported in yeast system or EMSA analysis (Hyungin et al., 2000; Tsaihung et al., 2010; Zhang et al., 2015; Wang et al., 2016a; Zhang et al., 2017). No shifted bands were observed in EMSA of DRE/CRT (Fig. 4C). This result suggested that IbbZIP37 may only be an ABRE-dependent transcription factor. In conclusion, these results indicate that IbbZIP37 is a transcription activor specifically binding to the ABRE cis-element and may further mediate the expression of downstream stress-responsive genes.

Most bZIP group A TFs such as $b Z I P 36 / A R E B 1 / A B F 2$, bZIP37/ABF3 and bZIP38/AREB2/ABF4 are highly induced by $\mathrm{ABA}$, drought and salinity treatments in plant tissues (Uno et al., 2000; Furihata et al., 2006; Yoshida et al., 2010). In our current study, IbbZIP37 can be induced by various environmental stresses. The change of transcription levels and response times showed that IbbZIP37 is very sensitive to ABA, PEG, salt and heat shock (Fig. 6). Our data thus further indicate that IbbZIP37 likely plays a positive role as an ABA-responsive transcription factor in abiotic stress signaling.

In conclusion, we report herein the transcription factor IbbZIP37 which contain the typical bZIP domain and conserved Ser/Thr kinase phosphorylation sites (RXXS/T) and can specifically bind to the ABRE cis-element. IbbZIP37 gene expression was induced with $\mathrm{ABA}$ and several environmental stresses including drought, salt and heat shock. The stress tolerance of the IbbZIP37-overexpresing plants remains to be further analyzed. Taken together, these current results broaden our understanding of sweetpotato bZIP TFs, and may offer an excellent candidate gene for improving stress tolerance in crop plants.

\section{ACKNOWLEDGMENTS}

This work was supported by the Basic Research Program of Shanxi Province (201801D121204), Cultivate Project of National Science Foundation of Shanxi Agricultural University (2017GPY06), Science Innovation Foundation of Shanxi Agricultural University (2018yz003) and Doctoral Research Grant of Shanxi Agricultural University (XB2009002) and KRIBB initiative program.

\section{Authors' contributions}

Study concepts and design: W. W. S. S. K.; Material preparation: W. W. X. Q. Y. Y. H. Y.; Literature research: W. W. Y. Y. H. S. K.; Date analysis/interpretation, Manuscript Preparation and definition of intellectual content: W. W. X. J. H. S. K.; Manuscript editing W. W. X. J. H. S. K.; Manuscript revision/review S. S. K. Manuscript final version approval W. W. X. Q. Y. Y. H. S. K. X. J. H. Y. S. S. K.; All authors read and approved the manuscript.

\section{REFERENCES}

Busk, P. K. and M. Pagès. 1998. Regulation of abscisic acid-induced transcription. Plant Mol. Biol. 37(3): 425-435.

Casaretto, J. A. and T. H. Ho. 2005. Transcriptional regulation by abscisic acid in barley (Hordeum vulgare L.) seeds involves autoregulation of the transcription factor HvABI5. Plant Mol. Biol. 57(1): 21-34.

Corrêa, L. G., D. M. Riaño-Pachón, C. G. Schrago, R. V. dos Santos, B. Mueller-Roeber and M. Vincentz. 2008. The role of bZIP transcription factors in green plant evolution: Adaptive features emerging from four founder genes. PLoS One. 3(8): e2944.

Curtis, M. D. and U. Grossniklaus. 2003. A gateway cloning vector set for high-throughput functional analysis of genes in planta. Plant Physiol. 133(2): 462-469.

Duvernay, W. H., M. S. Chinn and G. C. Yencho. 2013. Hydrolysis and fermentation of sweetpotatoes for production of fermentable sugars and ethanol. Ind. Crops Prod. 42(3): 527-537.

Fujii, H., V. Chinnusamy, A. Rodrigues, S. Rubio, R. Antoni, S. Y. Park, S. R. Cutler, J. Sheen, P. L. Rodriguez and J. K. Zhu. 2009. In vitro reconstitution of an abscisic acid signalling pathway. Nature. 462: 660-664.

Fujita, Y., M. Fujita, R. Satoh, K. Maruyama, M. M. Parvez, M. Seki, K. Hiratsu, M. Ohmetakagi, K. Shinozaki and K. Yamaguchishinozaki. 2005. AREB1 Is a transcription activator of novel ABRE-dependent ABA signaling that enhances drought stress tolerance in arabidopsis. Plant Cell. 17(12): 3470-3488.

Furihata, T., K. Maruyama, Y. Fujita, T. Umezawa, R. Yoshida, K. Shinozaki and K. Yamaguchishinozaki. 2006. Abscisic aciddependent multisite phosphorylation regulates the activity of a 
transcription activator AREB1. Proc. Natl. Acad. Sci. U. S. A. 103(6): 1988-1993.

García, M. N. M., V. Giammaria, C. Grandellis, M. T. Télleziñón, R. M. Ulloa and D. A. Capiati. 2012. Characterization of StABF1, a stress-responsive bZIP transcription factor from Solanum tuberosum L. That is phosphorylated by StCDPK2 in vitro. Planta. 235(4): 761-778.

Golldack, D., I. Lüking and O. Yang. 2011. Plant tolerance to drought and salinity: Stress regulating transcription factors and their functional significance in the cellular transcriptional network. Plant Cell Rep. 30(8): 1383-1391.

Goo, Y.M., E. H. Han, J. C. Jeong, S. S. Kwak, J. Yu, Y. H. Kim, M. J. Ahn and S. W. Lee. 2015. Overexpression of the sweet potato $\mathrm{lbOr}$ gene results in the increased accumulation of carotenoid and confers tolerance to environmental stresses in transgenic potato. C. R. Biol. 338(1): 12-20.

Hirayama, T. and K. Shinozaki. 2010. Research on plant abiotic stress responses in the post-genome era: Past, present and future. Plant J. Cell Mol. Biol. 61(6): 1041-1052.

Hossain, M., Y. Lee, J. Cho, C. Ahn, S. Lee, J. Jeon, H. Kang, C. Lee, G. An and P. Park. 2010a. The bZIP transcription factor OsABF1 is an ABA responsive element binding factor that enhances abiotic stress signaling in rice. Plant Mol. Biol. 72: 557-566.

Hossain, M. A., J. I. Cho, M. Han, C. H. Ahn, J. S. Jeon, G. An and P. B. Park. 2010b. The ABRE-binding bZIP transcription factor OsABF2 is a positive regulator of abiotic stress and ABA signaling in rice. J. Plant Physiol. 167(17): 1512-1520.

Hyungin, C., J. H. Hong, H. Jinok, J. Y. Kang and K. Sooyoung. 2000. ABFs, a family of ABA-responsive element binding factors. J. Biol. Chem. 275(3): 1723-1730.

Jakoby, M., B. Weisshaar, W. Drögelaser, J. Vicentecarbajosa, J. Tiedemann, T. Kroj and F. Parcy. 2002. BZIP transcription factors in arabidopsis. Trends Plant Sci. 7(3): 106-111.

Jin, R., B. H. Kim, C. Y. Ji, H. S. Kim, H. M. Li, D. F. Ma and S. S. Kwak. 2017. Overexpressing IbCBF3 increases low temperature and drought stress tolerance in transgenic sweetpotato. Plant Physiol. Biochem. 118: 45-54.

Kang, J. Y., H. I. Choi, M. Y. Im and S. Y. Kim. 2002. Arabidopsis basic leucine zipper proteins that mediate stress-responsive abscisic acid signaling. Plant Cell. 14(2): 343-357.

Kang, L., C. Y. Ji, S. H. Kim, Q. Ke, S. C. Park, H. S. Kim, H. U. Lee, J. S. Lee, W. S. Park, M. J. Ahn, H. S. Lee, X. P. Deng and S. S. Kwak. 2017a. Suppression of the $\beta$-carotene hydroxylase gene increases $\beta$-carotene content and tolerance to abiotic stress in transgenic sweetpotato plants. Plant Physiol. Biochem. 117: 24-31.

Kang, L., H. S. Kim, Y. S. Kwon, Q. Ke, C. Y. Ji, S. C. Park, H. S. Lee, X. Deng and S. S. Kwak. 2017b. IbOr regulates photosynthesis under heat stress by stabilizing ibpsbp in sweetpotato. Front. Plant Sci. 8: 989.

Kim, H. S., S. C. Park, Y. J. Chang, S. Park, J. C. Jeong, H. S. Lee and S. S. Kwak. 2016. Molecular characterization of biotic and abiotic stress-responsive MAP kinase genes, IbMPK3 and IbMPK6, in sweetpotato. Plant Physiol. Biochem. 108: 37-48.

Kim, S. H., J. C. Jeong, S. Park, J. Y. Bae, M. J. Ahn, H. S. Lee and S. S. Kwak. 2014. Down-regulation of sweetpotato lycopene $\hat{I}^{2}$-cyclase gene enhances tolerance to abiotic stress in transgenic calli. Mol. Biol. Rep. 41(12): 8137-8148.

Kobayashi, F., E. Maeta, A. Terashima and S. Takumi. 2008. Positive role of a wheat HVABI5 ortholog in abiotic stress response of seedlings. Physiol. Plant. 134(1): 74-86.

Latchman, D. S. 1997. Transcription factors: An overview. Int. J. Exp. Pathol. 74(5): 1305-1312.
Lebot, V. 2010. Sweet potato. In: J. E. Bradshaw (Ed.), Root and Tuber Crops, Handbook of Plant Breeding, Ch. 3. Springer, New York, p. 97-125.

Lindemose, S., C. O'Shea, M. K. Jensen and K. Skriver. 2013. Structure, function and networks of transcription factors involved in abiotic stress responses. Int. J. Mol. Sci. 14(3): 5842-5878.

Madzlan, K., H. Hasnisa, S. Sabeetha and M. N. Dayana. 2012. Extraction of starch and enzymatic production of high amylose starch from sweetpotato (Ipomea batatas) var. Telong. J. Trop. Agric. Food Sci. 222: 203-210.

Muszynski, M. G., T. Dam, B. Li, D. M. Shirbroun, Z. Hou, E. Bruggemann, R. Archibald, E. V. Ananiev and O. N. Danilevskaya. 2006. Delayed flowering1 encodes a basic leucine zipper protein that mediates floral inductive signals at the shoot apex in maize. Plant Physiol. 142(4): 1523-1536.

Pradhan, D. M. P., A. Mukherjee, J. George, S. K. Chakrabarti, B. Vimala, S. K. Naskar, B. K. Sahoo and S. Samal. 2015. High starch, beta carotene and anthocyanin rich sweet potato: Ascent to future food and nutrition security in coastal and backward areas. Int. J. Trop. Agric. 10(1): 9-22.

Roychoudhury, A., S. Paul and S. Basu. 2013. Cross-talk between abscisic acid-dependent and abscisic acid-independent pathways during abiotic stress. Plant Cell Rep. 32(7): 985-1006.

Sun, H. K., Y. O. Ahn, M. J. Ahn, J. C. Jeong, H. S. Lee and S. S. Kwak. 2013. Cloning and characterization of an orange gene that increases carotenoid accumulation and salt stress tolerance in transgenic sweetpotato cultures. Plant Physiol. Biochem. 70(1): 445-454.

Thurow, C., A. Schiermeyer, S. Krawczyk, T. Butterbrodt, K. Nickolov and C. Gatz. 2005. Tobacco bZIP transcription factor TGA2.2 and related factor TGA2.1 have distinct roles in plant defense responses and plant development. Plant J. 44(1): 100-113.

Tsaihung, H., C. W. Li, R. C. Su, C. P. Cheng, T. Yichien and M. T. Chan. 2010. A tomato bZIP transcription factor, SLAREB, is involved in water deficit and salt stress response. Planta. 231(6): 1459-1473.

Uno, Y., T. Furihata, H. Abe, R. Yoshida, K. Shinozaki and K. Yamaguchi-Shinozaki. 2000. Arabidopsis basic leucine zipper transcription factors involved in an abscisic acid-dependent signal transduction pathway under drought and high-salinity conditions. Proc. Natl. Acad. Sci. U. S. A. 97(21): 11632-11637.

Wang, J., Q. Li, X. Mao, A. Li and R. Jing. 2016a. Wheat transcription factor TaAREB3 participates in drought and freezing tolerances in arabidopsis. Int. J. Biol. Sci. 12(2): 257-269.

Wang, W., B. Vinocur and A. Altman. 2003. Plant responses to drought, salinity and extreme temperatures: Towards genetic engineering for stress tolerance. Planta. 218(1): 1-14.

Wang, Z., G. Su, M. Li, Q. Ke, S. Y. Kim, H. Li, J. Huang, B. Xu, X. P. Deng and S. S. Kwak. 2016b. Overexpressing arabidopsis ABF3 increases tolerance to multiple abiotic stresses and reduces leaf size in alfalfa. Plant Physiol. Biochem. 109: 199-208.

Xiang, Y., N. Tang, H. Du, H. Ye and L. Xiong. 2008. Characterization of OsbZIP23 as a key player of the basic leucine zipper transcription factor family for conferring abscisic acid sensitivity and salinity and drought tolerance in rice. Plant Physiol. 148(4): 1938-1952.

Yamaguchishinozaki, K. and K. Shinozaki. 2005. Organization of cis-acting regulatory elements in osmotic- and cold-stressresponsive promoters. Trends Plant Sci. 10(2): 88-94.

Yamaguchishinozaki, K. and K. Shinozaki. 2006. Transcriptional regulatory networks in cellular responses and tolerance to dehydration and cold stresses. Ann. Rev. Plant Biol. 57(57): 781-803. 
Yoshida, T., Y. Fujita, H. Sayama, S. Kidokoro, K. Maruyama, J. Mizoi, K. Shinozaki and K. Yamaguchi-Shinozaki. 2010. AREB1, AREB2, and ABF3 are master transcription factors that cooperatively regulate $A B R E-d e p e n d e n t A B A$ signaling involved in drought stress tolerance and require $A B A$ for full activation. Plant J. 61(4): 672-685.

Zhang, L., L. Zhang, C. Xia, L. Gao, C. Hao, G. Zhao, J. Jia and
X. Kong. 2017. A novel wheat C-bZIP gene,TabZIP14-B, participates in salt and freezing tolerance in transgenic plants. Front. Plant Sci. 8: 710.

Zhang, L., L. Zhang, C. Xia, G. Zhao, J. Liu, J. Jia and X. Kong. 2015. A novel wheat bZIP transcription factor, TabZIP60, confers multiple abiotic stress tolerances in transgenic Arabidopsis. Physiol. Plant. 153(4): 538-554 